\title{
Dosimetric Comparison of Fixed and Flexible Geometry ICRT Applicators in Patients Treated for Carcinoma Cervix
}

\author{
Sukhvir Singh, Arti Sarin, Manoj Kumar Semwal*, Ajay Kumar Singh, Pankaj Goyal, \\ Ashok Kumar \\ Department of Radiation Oncology, Army Hospital (R\&R), Delhi Cantonment, New Delhi, India \\ Email: " manojsemwal@yahoo.co.in
}

Received 22 December 2014; accepted 6 January 2015; published 16 January 2015

Copyright (C) 2015 by authors and Scientific Research Publishing Inc.

This work is licensed under the Creative Commons Attribution International License (CC BY). http://creativecommons.org/licenses/by/4.0/

(c) () Open Access

\begin{abstract}
In intracavitary radiotherapy (ICRT) for cancer cervix, applicator geometry has the potential to impact the doses to organs at risk (OARs) and the coverage of the target volume. At our centre two Varian made Fletcher-style applicator sets, namely defined (fixed) geometry and flexible geometry, are used for ICRT. In the present work, the two types of applicators were compared dosimetrically as per the recommendations of the International Commission on Radiation Units and Measurements (ICRU-38) while delivering high dose rate brachytherapy. Twenty four patients who underwent ICRT were considered for this study. Radiographic method was used for treatment planning on Abacus planning system. ICRU-38 recommended parameters were estimated and compared. Flexible geometry applicator showed $8.8 \%$ and $16 \%$ higher bladder and rectum point doses as compared to the fixed geometry one but the difference was statistically not significant. The thickness of the pear-shaped isodose volume was larger for the flexible geometry implant also indicating towards higher doses to OARs. The higher bladder and rectum point doses in the case of flexible applicator will need to be validated by a larger data set.
\end{abstract}

\section{Keywords}

Carcinoma Cervix, Intracavitary Radiotherapy, Applicator Geometry, Dosimetry

\section{Introduction}

Intracavitary radiotherapy (ICRT) is the most widely used form of brachytherapy in the treatment of cancer cervix.

"Corresponding author.

How to cite this paper: Singh, S., Sarin, A., Semwal, M.K., Singh, A.K., Goyal, P. and Kumar, A. (2015) Dosimetric Comparison of Fixed and Flexible Geometry ICRT Applicators in Patients Treated for Carcinoma Cervix. International Journal of Medical Physics, Clinical Engineering and Radiation Oncology, 4, 1-6. http://dx.doi.org/10.4236/ijmpcero.2015.41001 
The technique and technology of ICRT have evolved over a century to the present state. The early preloaded applicators such as the Manchester type one having rubber tandems and cork ovoids, free from one other, gave way to manual afterloading ones which in turn have been substituted by remote afterloading high dose rate (HDR) applicators. Fletcher and Suit designed the first afterloading ICRT applicator in the 1960s. Various brachytherapy equipment manufacturers depending on the existing designs and feed-back from the users have come out with modifications on these designs. Any applicator design, due to its specific geometric features, offers isodose shapes that are somewhat different from other types of applicators.

M/s Varian Medical Systems commercially offer many types of applicators for ICRT. Our centre possesses two types of Varian make ICRT applicators namely Fletcher-style applicator set with defined (fixed) geometry, and Fletcher-style applicator set with flexible geometry for Gammamed 12i HDR Brachytherapy machines (Figure 1). Both these applicator types are based on the dosimetry of the conventional Fletcher-style application. However, the relative positions of the ovoids with respect to the tandem are different in both these sets as evident in the lateral radiographs shown in Figure 2. The colpostats are positioned more posteriorly in the flexible design as compared to the fixed one. It is believed that this difference in relative positions of the tandem and colpostats may have implications on the treated volume and sparing of the organs at risk especially in situations where 3-dimensional image based treatment planning and optimization is not carried out [1]-[3].

Of late more and more centres are moving towards 3-D image-based planning in brachytherapy. And for this purpose the guidelines issued by the Image-Guided Brachytherapy Working Group of the American Brachytherapy Society, and the Groupe European de Curethérapie-European Society for Therapeutic Radiology and Oncology (GEC-ESTRO) working group, have been widely accepted [4]-[6]. However, a large number of centres practicing brachytherapy in India still use 2D orthogonal film based dosimetry for ICRT. A few centres due to heavy patient load are not in a position to carry out even 2D film based planning for all the patients. These centres practice standard applicator geometry, as stored in their treatment planning system library, based on treatment planning. It has been established by many studies that the 2D system of point dose prescription and reporting based on orthogonal radiographic reconstruction of applicators has limitations as compared to 3D volume dose prescription and reporting in terms of dose estimation to OARs and treated volume estimation [7][10].

At our centre, due to non-availability of CT/MRI based planning resources, we follow 2D orthogonal radiograph based planning with dose prescription at Manchester point A. We estimate dose to bladder and rectum as per International Commission on Radiation Units and Measurements (ICRU) definition (ICRU-38) and proceed with the treatment delivery only when the organ at risk (OAR) doses are considered safe [11]. The comprehensive ICRU-38 recommendations that include additional points for estimation of dose, dimensions of the pearshaped volume formed by the prescription isodose, and total reference air kerma (TRAK) values are not estimated for all the cases.

There have been a few comparative dosimetry studies on ICRT applicators reported in literature [12]-[14]. However, we found no such study involving patients treated with the types of ICRT applicators used at our centre. It was felt that the geometry of the flexible applicator system seemingly has the potential of increasing the dose to the rectum. Therefore, we decided to carry out a comparative study of the impact of the two designs on the patient dosimetric parameters as recommended by ICRU-38. Data of a total of 24 ICRT applications performed on 24 patients were considered for this comparison. The present study only compares physical doses and related parameters without consideration of the radiobiological factors. It is believed that this study will be useful for clinicians in deciding the type of applicators depending on the clinical requirement.

\section{Material and Methods}

Patients: At this centre all advanced stage cervical cancer patients are considered for brachytherapy following external beam radiotherapy (EBRT). Eligibility requirements for patient inclusion for brachytherapy are: minimum haemoglobin 10 gm, performance status 70 or more (Karnofsky Scale), and histopathological confirmation of the disease. All patients are evaluated for brachytherapy after completion of EBRT. The protocol for radiotherapy is EBRT using a $15 \mathrm{MV}$ photon beam from a linear accelerator to a dose of 5040 cGy in 28 conventional fractions. This followed by ICRT to a dose of 21 Gy delivered in three weekly fractions prescribed to point A. For the present study, orthogonal films and other ICRT records of twenty four patients were randomly selected; of them ten were with flexible applicator and 14 with fixed applicator. 


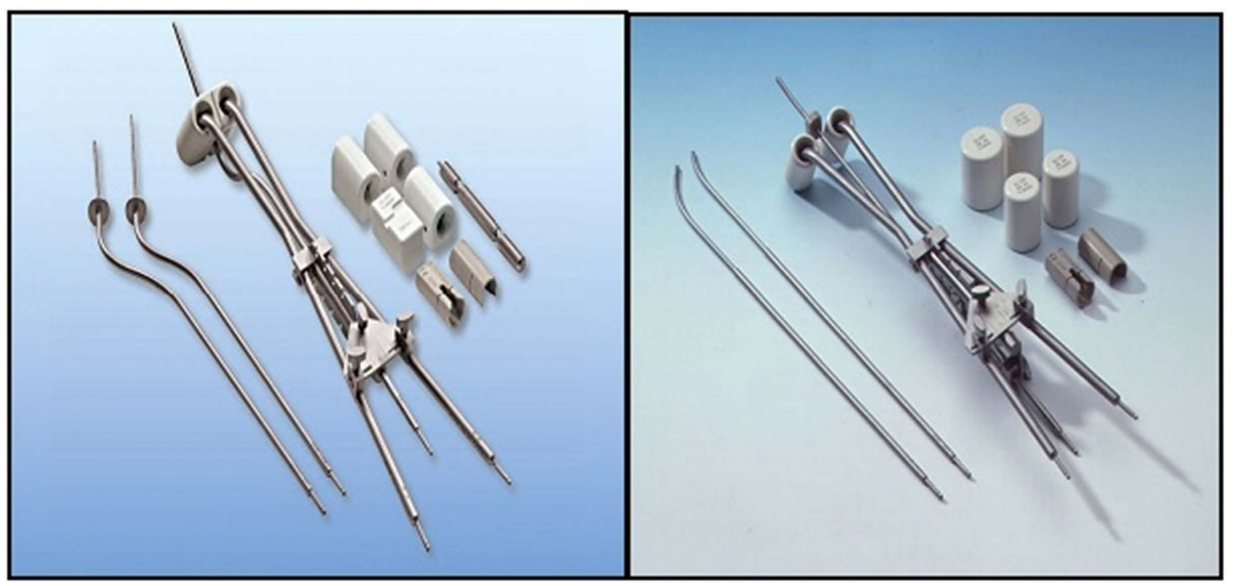

(a)

(b)

Figure 1. Varian applicators: (a) Defined (fixed) geometry Fletcher-style applicator set; (b) Flexible geometry Fletcher-style applicator set (figure taken from Varian technical catalogue).

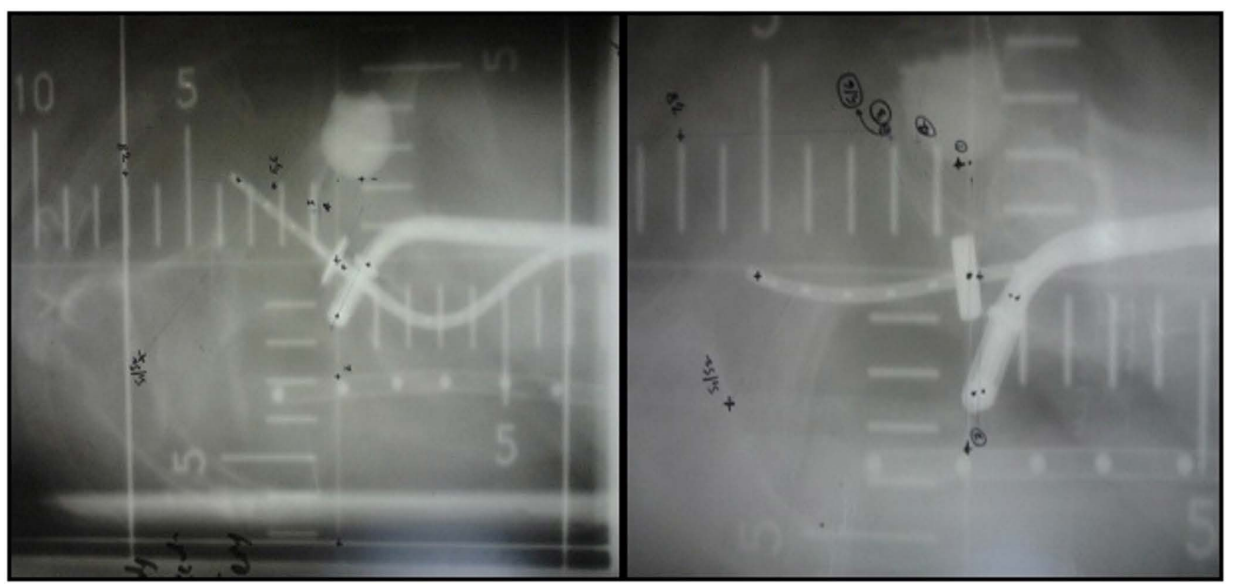

(a)

(b)

Figure 2. Lateral radiographs of two typical ICRT implants (a) with defined (fixed) geometry Fletcher-style applicator (b) with flexible geometry Fletcher-style applicator. The Foley’s balloon and the rectum marker along with the applicator probes are visible in the radiographs.

Applicators and Equipment: The treatment planning system used was ABACUS (version 3.1 Release 5) from M/s Varian. The Fletcher-style applicator set with defined (fixed) geometry (part no. GM11000810), and the Fletcher-style applicator set with flexible geometry (part no. GM11000730) were used for ICRT (Figure 1). The shape and size of the ovoids (colpostats) is the same in both the types. The source probe in these colpostats makes a 60 degree angle with the vaginal axis. The three intrauterine probes of the fixed geometry set are curved $30^{\circ}$ in relation to vaginal axis and have fixed lengths of 4,6 , and $8 \mathrm{~cm}$ with permanent stopper/flange at these lengths. In the case of flexible geometry applicators, the probes are curved $15^{\circ}, 30^{\circ}$ or $45^{\circ}$ in relation to the vaginal axis and have no fixed lengths. The stopper/flange can be fixed at lengths as per the patient anatomy unlike the fixed geometry type. It is evident that the intrauterine tandem probe will make angles 75, 90 or 105 degree with respect to the colpostats probes in the case of flexible geometry type applicator system depending on the tandem probe angle chosen whereas the angle will be 90 degree for all probe combinations in the case of fixed geometry applicators. Orthogonal radiographs are taken on a simulator model Simview 3000 from Siemens and Gammmed 12i HDR brachytherapy machine from Varian is used for treatment delivery.

Brachytherapy procedure and treatment planning: Patient's recto-vaginal bimanual examination and subsequent ICRT applicator placement is done under general anaesthesia. First a Foley balloon catheter is inserted into the bladder, and the balloon is filled with 7 cc of diluted radio-opaque contrast solution. The length of the 
tandem to be inserted into the uterus is determined with a uterine sound. Depending upon the availability of the applicators either the fixed geometry or the flexible geometry applicator is chosen. The flange abuts the external os (EOS), restricting further superior movement of the tandem and thus preventing uterine perforation. The ovoids are positioned within the vaginal vault in the lateral cervical fornices. The largest ovoids allowed by the anatomy were used for better dosimetric results. The tandem and ovoids are immobilized with gauze packing, which also aided in sparing of rectum and bladder. For rectum delineation a flexible rectal marker tube with outer diameter about $6 \mathrm{~mm}$ and having radiopaque beads within it is used.

On the orthogonal radiographs, all the dosimetry points were digitized as per ICRU-38 recommendations in the ABCUS planning system for each of the selected patient. Equal-time optimization was carried out for a realistic comparison between the two systems. Applicator details such as type of applicator, length of the tandem, angle of the tandem, deviation of the tandem from midline, cranio-caudal distance between the flange and the tip of the ovoids were recorded. Using the ABACUS system dose to each ICRU point was estimated for a prescription dose of 7 Gy to point A. Also, dimension of the volume covered by prescription isodose, and TRAK were estimated.

\section{Results and Discussion}

It is generally considered that for an optimal implant geometry, the tandem should bisect the ovoids, and about $1 / 3$ of the ovoids should be superior to the lowest end of the tandem i.e. the flange. A look at the two types of applicators as shown in Figure 2 indicates that in the case of flexible type of applicator, the tandem does not bisect the ovoids. The ovoids seem to be reaching more in the posterior direction as compared to the fixed geometry applicator. Table 1 shows the doses to various reference points for the two types of applicators, TRAK value, and dimensions of the prescription isodose volume (7 Gy). The statistical analysis was carried out using WINKS SDA (version 6.0.9) programme for estimating p-values for t-test and analyses of variance (ANOVA).

It is observed that the average bladder dose is $8.8 \%$ higher (4.8 vs $5.2 \mathrm{~Gy}$, p-value 0.659), whereas average rectum dose is $16 \%$ higher (5.8 Gy vs $5.0 \mathrm{~Gy}$, p-value 0.254 ) in the case of flexible type applicator as compared to fixed geometry applicator. The difference though is statistically not significant in both the cases. Further, as seen in the table the thickness of the $7 \mathrm{~Gy}$ isodose surface is larger in the flexible geometry being $5 \mathrm{~cm}$ as compared to $4.5 \mathrm{~cm}$ for fixed geometry applicator, and the difference is statistically significant (p-value $<0.001)$. The thickness value for the fixed applicator is similar to the one quoted by Bansal et al., i.e. $4.45 \mathrm{~cm}$ [15]. The thickness difference in the two types of geometries could translate into higher rectum/bladder dose but was not found to be so for all the cases. The doses to these OARs (rectum and bladder) depend on a few other factors such as vaginal packing and lateral shift in the rectum marker and lateral/superior-interior positioning of the Foley's balloon with respect to the ovoids and tandem for each patient. As mentioned earlier, the implant geometries as shown in Figure 2(a) and Figure 2(b) indicate toward higher dose in rectum for the flexible implant; the rectum point being closer to the ovoid sources. It is, therefore, felt that though the data shows the trend towards higher rectum dose, as was also expected from the implant geometry, yet due to smallness of the sample size and other anatomical factors the trend is statistically not significant. Maybe a larger patient data set is required to validate the findings.

As regards doses to other ICRU points, it is observed that the difference, if any, among the two types of applicators is small. That means dose distributions at comparatively larger distances from the applicator probes are almost similar for the two types of applicator geometries. This is further confirmed by the comparison of the dimensions of the 7 Gy volumes shown in Table 1. The height and width of the 7 Gy volume is not different between the two categories of implants. The TRAK values are also similar in both the cases as shown in Table 1. These values (4.91 and $4.93 \mathrm{mGy}$ ) are close to the value (5.1 mGy) reported by Bansal et al. (2014) [15]. The tilt angle of the intrauterine tandem towards left or right side with respect to the patient midline was also noted to its effect on dose distribution. The maximum tilt noted was about 8 degree. Even with this tilt value we did not find differences in doses on left or right side ICRU points. Maybe other factors such as spacing of ovoids, distance between the flange and the ovoid mid position were also influencing the point doses on the ICRU trapezoid.

\section{Conclusion}

This study was initiated to estimate the dosimetric consequences of differences in the geometries of the two types of ICRT applicators in terms of relative positions of the tandem and the colpostats supplied by M/s Varian. 
Table 1. ICRU-38 recommended patient dosimetric parameters for the two types of applicators.

\begin{tabular}{|c|c|c|c|c|c|c|}
\hline \multirow{3}{*}{ ICRU Parameters } & \multicolumn{3}{|c|}{ Flexible Geometry Applicator (tandem length in cm) } & \multicolumn{3}{|c|}{ Fixed Geometry Applicator (tandem length in cm) } \\
\hline & 4.0 & 6.0 & Combined & 4.0 & 6.0 & Combined \\
\hline & \multicolumn{6}{|c|}{ Dose in Gy (mean \pm std dev) } \\
\hline BP & $5.37 \pm 1.20$ & $4.95 \pm 1.09$ & $5.16 \pm 1.11$ & $5.02 \pm 2.24$ & $4.99 \pm 3.22$ & $4.83 \pm 2.62$ \\
\hline $\mathrm{RP}$ & $6.51 \pm 1.46$ & $5.01 \pm 1.12$ & $5.76 \pm 1.46$ & $4.56 \pm 1.43$ & $5.50 \pm 1.16$ & $5.03 \pm 1.53$ \\
\hline LPW & $1.32 \pm 0.26$ & $1.51 \pm 0.32$ & $1.41 \pm 0.29$ & $1.74 \pm 0.33$ & $1.62 \pm 0.18$ & $1.68 \pm 0.26$ \\
\hline RPW & $1.34 \pm 0.33$ & $1.50 \pm 0.16$ & $1.42 \pm 0.258$ & $1.35 \pm 0.20$ & $1.50 \pm 0.25$ & $1.42 \pm 0.23$ \\
\hline LEI & $1.17 \pm 0.14$ & $1.48 \pm 0.34$ & $1.32 \pm 0.29$ & $1.49 \pm 0.03$ & $1.41 \pm 0.23$ & $1.45 \pm 0.16$ \\
\hline REI & $1.19 \pm 0.15$ & $1.19 \pm 0.09$ & $1.19 \pm 0.12$ & $1.10 \pm 0.11$ & $1.31 \pm 0.14$ & $1.20 \pm 0.16$ \\
\hline LCI & $0.59 \pm 0.07$ & $1.19 \pm 0.34$ & $0.89 \pm 0.39$ & $0.82 \pm 0.13$ & $1.24 \pm 0.48$ & $1.03 \pm 0.39$ \\
\hline RCI & $0.61 \pm 0.09$ & $0.93 \pm 0.19$ & $0.77 \pm 0.22$ & $0.69 \pm 0.13$ & $1.25 \pm 0.38$ & $0.97 \pm 0.39$ \\
\hline LP & $0.19 \pm 0.04$ & $0.34 \pm 0.08$ & $0.264 \pm 0.10$ & $0.26 \pm 0.04$ & $0.42 \pm 0.12$ & $0.33 \pm 0.12$ \\
\hline RP & $0.19 \pm 0.04$ & $0.32 \pm 0.08$ & $0.256 \pm 0.09$ & $0.25 \pm 0.05$ & $0.44 \pm 0.12$ & $0.34 \pm 0.13$ \\
\hline \multicolumn{7}{|c|}{7 Gy Isodose Volume (dimensions in cm) } \\
\hline Height & $7.01 \pm 0.42$ & $8.25 \pm 0.22$ & $7.63 \pm 0.72$ & $7.48 \pm 0.27$ & $9.30 \pm 0.15$ & $8.39 \pm 0.97$ \\
\hline Width & $4.97 \pm 0.41$ & $4.72 \pm 0.19$ & $4.85 \pm 0.33$ & $5.36 \pm 0.12$ & $5.27 \pm 0.30$ & $5.32 \pm 0.22$ \\
\hline Thickness & $4.95 \pm 0.22$ & $5.08 \pm 0.20$ & $5.01 \pm 0.21$ & $4.63 \pm 0.18$ & $4.42 \pm 0.26$ & $4.53 \pm 0.232$ \\
\hline \multicolumn{7}{|c|}{ TRAK (mGy at 1 meter) } \\
\hline TRAK & $4.74 \pm 0.26$ & $5.08 \pm 0.23$ & $4.91 \pm 0.29$ & $4.72 \pm 0.24$ & $5.13 \pm 0.16$ & $4.93 \pm 0.29$ \\
\hline
\end{tabular}

TRAK = total reference air kerma (mGy at 1 meter). $\mathrm{BP}=$ bladder point; $\mathrm{RP}=$ rectum point; $\mathrm{LPW}=$ left pelvic wall point; RPW = right pelvic wall point; REI = right external iliac point; LEI = left external iliac point; LCI = left common iliac point; RCI = right common iliac point; LP = left para-aortic point; RP = right para-aortic point.

The impact of these differences especially on rectum dose, which appeared closer to the applicators in the flexible-type geometry, would mean increased dose to the rectum. With the available radiographic method used in this study, we found that both the rectum and the bladder ICRU-38 point doses were higher in case of flexible implant, and there was no difference for the other ICRU-38 parameters. The data set will need to be expanded to statistically validate the results. Also, estimation of differences in terms of coverage of clinical target volume, and volumetric OAR doses that is clinically more meaningful will require image based 3D treatment planning. Brachytherapy including ICRT as part of radiotherapy will continue to play a significant role in managing cancer cervix. Future technological innovations in this area will focus on further improving the accuracy of dose delivery to the tumour and sparing of the normal organs.

\section{References}

[1] Rutten, R.R., Lawyer, A.A. and Berner, P. (1998) Dose Variation Due to Differences in Applicator Placement Used for Intracavitary Brachytherapy of Cervical Cancer. Medical Dosimetry, 23, 57-63. http://dx.doi.org/10.1016/S0958-3947(97)00110-6

[2] Chakraborty, S., Patel, F.D., Patil, V.M., Oinam, A.S. and Sharma, S.C. (2014) Magnitude and Implications of Interfraction Variations in Organ Doses during High Dose Rate Brachytherapy of Cervix Cancer: A CT Based Planning Study. ISRN Oncology, Article ID: 687365

[3] Datta, N.R., Kumar, S., Das, K.J., Pandey, C.M., Halder, S. and Ayyagari, S. (2001) Variations of Intracavitary Applicator Geometry during Multiple HDR Brachytherapy Insertions in Carcinoma Cervix and Its Influence on Reporting as per ICRU Report 38. Radiotherapy \& Oncology, 60, 15-24. http://dx.doi.org/10.1016/S0167-8140(01)00352-8

[4] Nag, S., Cardenes, H., Chang, S., Das, I.J., Erickson, B., Ibbott, G.S., et al. (2004) Image-Guided Brachytherapy Working Group. Proposed Guidelines for Image-Based Intracavitary Brachytherapy for Cervical Carcinoma. International Journal of Radiation Oncology • Biology • Physics, 60, 1160-1172.

http://dx.doi.org/10.1016/j.ijrobp.2004.04.032 
[5] Haie-Meder, C., Potter, R., Van Limbergen, E., Briot, E., De Brabandere, M., Dimopoulos, J., et al. (2005) Recommendations from Gynaecological (GYN) GEC ESTRO Working Group (I): Concepts and Terms in 3D Image Based 3D Treatment Planning in Cervix Cancer Brachytherapy with Emphasis on MRI Assessment of GTV and CTV. Radiotherapy \& Oncology, 74, 235-245. http://dx.doi.org/10.1016/j.ijrobp.2004.04.032

[6] Potter, R., Haie-Meder, C., Limbergen, E.V., Barillot, I., Brabandere, M.D., Dimopoulos, J., et al. (2006) Recommendations from Gynaecological (GYN) GEC ESTRO Working Group (II): Concepts and Terms in 3D Image-Based Treatment Planning in Cervix Cancer Brachytherapy-3D Dose Volume Parameters and Aspects of 3D Image-Based Anatomy, Radiation Physics, Radiobiology. Radiotherapy \& Oncology, 78, 67-77. http://dx.doi.org/10.1016/j.radonc.2005.11.014

[7] Fellner, C., Potter, R., Knocke, T.H. and Wambersie, A. (2001) Comparison of Radiography- and Computed Tomography Based Treatment Planning in Cervix Cancer in Brachytherapy with Specific Attention to Some Quality Assurance Aspects. Radiotherapy \& Oncology, 58, 53-62. http://dx.doi.org/10.1016/S0167-8140(00)00282-6

[8] Addeo, D., Duckworth, T., Blank, S., Hitchen, C., Donach, M. and Formenti, S. (2008) Correlation between (ICRU) Point Doses and CT Based Image Planning of Intracavitary Brachytherapy for Cervical Cancer. Proceedings of the 50th Annual ASTRO Meeting. International Journal of Radiation Oncology • Biology • Physics, 72, S374. http://dx.doi.org/10.1016/j.ijrobp.2008.06.1223

[9] Wachter-Gerstner, N., Wachter, S., Reinstadler, E., Fellner, C., Knocke, T.H., Wambersie, A. and Potter, R. (2003) Bladder and Rectum Dose Defined from MRI Based Treatment Planning for Cervix Cancer Brachytherapy: Comparison of Dose-Volume Histograms for Organ Contours and Organ Wall, Comparison with ICRU Rectum and Bladder reference Points. Radiotherapy \& Oncology, 68, 269-276. http://dx.doi.org/10.1016/S0167-8140(03)00189-0

[10] Jamema, S.V., Saju, S., Mahantshetty, U., Pallad, S., Deshpande, D.D., Shrivastava, S.K., et al. (2008) Dosimetric Evaluation of Rectum and Bladder Using Image-Based CT Planning and Orthogonal Radiographs with ICRU 38 Recommendations in Intracavitary Brachytherapy. Journal of Medical Physics, 33, 3-8. http://dx.doi.org/10.4103/0971-6203.39417

[11] International Commission on Radiation Units and Measurements (1985) Dose and Volume Specification for Reporting Intracavitary Therapy in Gynaecology. ICRU Report 38, ICRU, Bethesda.

[12] Thirion, P., Kelly, C., Salib, O., Moriarty, M., O’Reilly, D., Griffin. M., et al. (2005) A Randomized Comparison of Two Brachytherapy Devices for the Treatment of Uterine Cervical Carcinoma. Radiotherapy \& Oncology, 74, $247-250$. http://dx.doi.org/10.1016/j.radonc.2004.10.018

[13] Masterson, M.E., Thomason, C., Hunt, M., Anderson, L., Eisenbarth, J. and Belanich, M. (1982) A Comparative Study of Henschke and Fletcher-Suit Cervical Applicators Using Cs-137. International Journal of Radiation Oncology • Biology $\bullet$ Physics, 8, 89.

[14] Nath, R., Urdaneta, N., Bolanis, N. and Peschel, R. (1991) A Dosimetric Analysis of Moris Fletcher, and Henschke Systems for Treatment of Uterine Cervix Carcinoma. International Journal of Radiation Oncology • Biology • Physics, 21, 995-1003. http://dx.doi.org/10.1016/0360-3016(91)90741-L

[15] Bansal, A.K., Semwal, M.K., Sharma, D.N., Thulkar, S., Julka, P.K. and Rath, G.K. (2014) A Patient Based Dosimetric Study of Intracavitary and Interstitial Brachytherapy in Advanced Stage Carcinoma of the Cervix. Journal of Applied Clinical Medical Physics, 15, 63-70. 
Scientific Research Publishing (SCIRP) is one of the largest Open Access journal publishers. It is currently publishing more than 200 open access, online, peer-reviewed journals covering a wide range of academic disciplines. SCIRP serves the worldwide academic communities and contributes to the progress and application of science with its publication.

Other selected journals from SCIRP are listed as below. Submit your manuscript to us via either submit@scirp.org or Online Submission Portal.
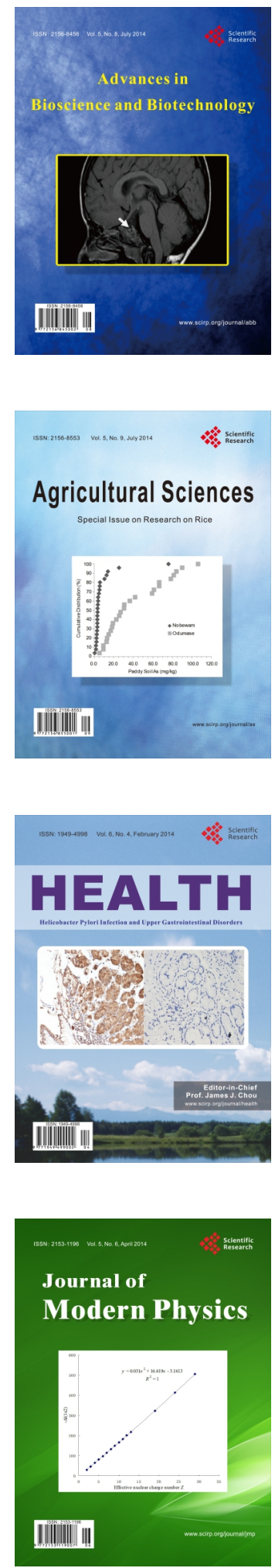
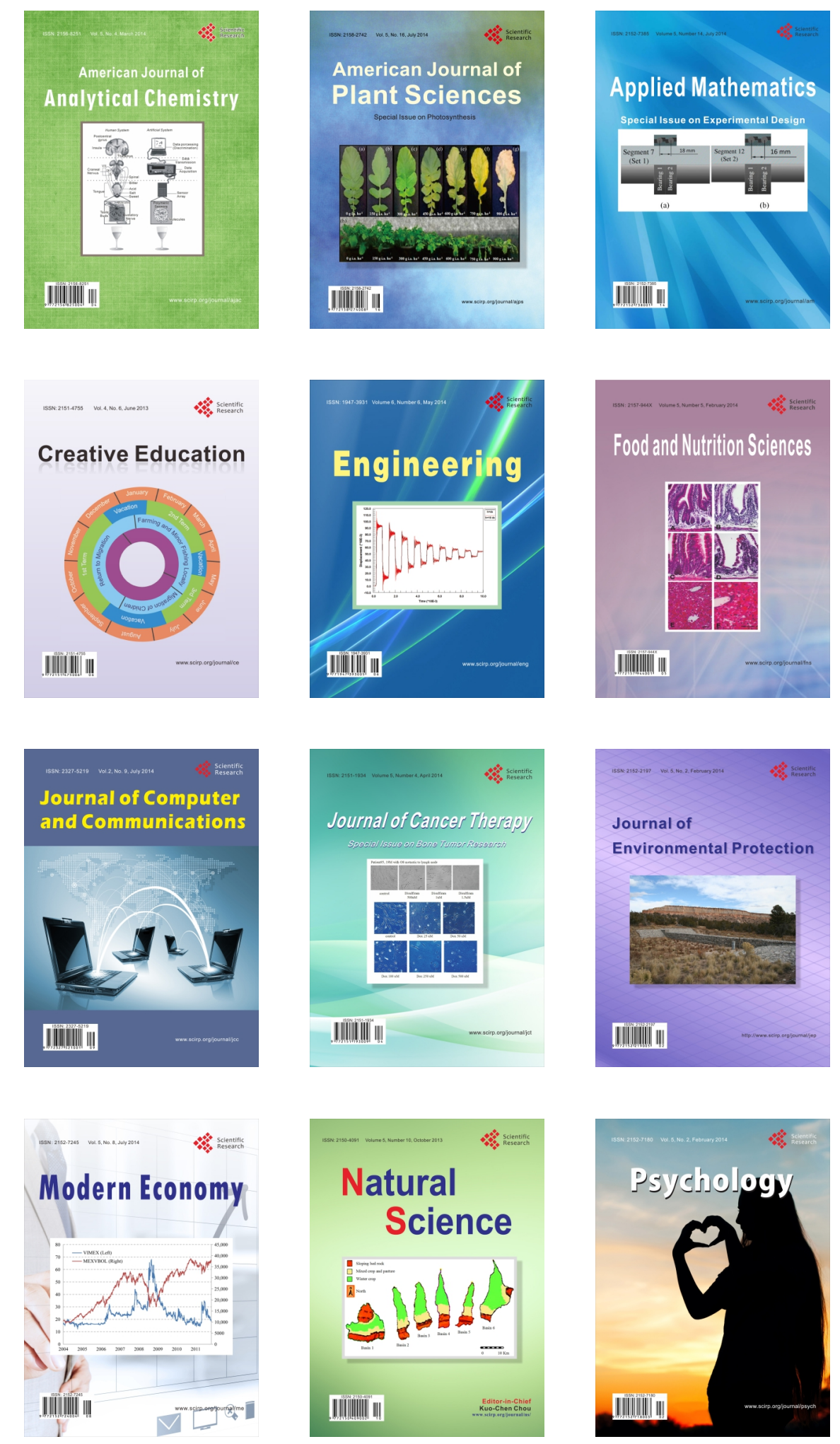